\title{
Two Cases of First Branchial Cleft Anomalies Medial to the Facial Nerve
}

\author{
Jeong Hwan Choi ${ }^{\mathrm{a}}$, Hoon Young Woo ${ }^{\mathrm{a}, \mathrm{b}}$
}

\begin{abstract}
First branchial cleft anomalies usually come to the clinician's attention because of an external opening or recurrent infections. For the safe and complete resection, a thorough understanding of the interrelationship of the anomaly to the facial nerve and identification of the facial nerve at the operative field is required. The risk of facial nerve injury increases in patients who have had multiple infections or surgical procedures. We present detailed figures demonstrating the relationship of the branchial cleft cysts to the facial nerve and emphasize that successful total resection without complications require exposure of the lesion's relationships to the facial nerve.
\end{abstract}

Keywords: First branchial cleft anomalies; Facial nerve

\section{Introduction}

First branchial cleft anomalies account for less than $8 \%$ of all branchial abnormalities [1]. The successful treatment of first branchial cleft anomalies requires understanding of their relationship to the facial nerve. First branchial cleft cyst runs

Manuscript accepted for publication January 19, 2012

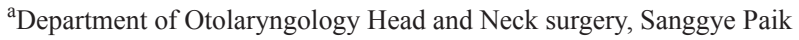

Hospital, College of Medicine, Inje University, Seoul, Korea

${ }^{\mathrm{b}}$ Corresponding author: Hoon Young Woo, Department of

Otorhinolaryngology, Inje University Sanggyepaik Hospital, 761-1

Sanggye-7-dong Nowon-gu, Seoul, 139-707, Republic of Korea (South).

Email: why@paik.ac.kr

doi: $10.4021 / \mathrm{jcs} 9 \mathrm{w}$ deep or superficial to the facial nerve. The cyst which is superficial to the facial nerve is easy to be excised. But, if it runs deep to the facial nerve, it would be resected with high possibility of neural damage. Surgery mandates complete resection and facial nerve preservation. Both of these goals are best accomplished by initial dissection and exposure of the facial nerve, followed by a safe excision of the anomaly.

\section{Case Report}

\section{Case 1}

A 20-year-old female presented to our clinic with the symptom of recurrent local swelling at the anterior border of the left sternocleidomastoid muscle. The area surrounding the mass was scarred due to previous infections and surgery. On examination, there was a small opening at the floor of the cartilaginous part of the left external auditory canal. A small fistulous opening was noted at the floor of the cartilaginous part of the left external auditory canal (Fig. 1A). Her tympanic membrane and rest her examination was normal. A modified Blair incision was performed to expose the facial nerve and its branches and to identify the relationship of the lesion to the facial nerve. During surgical exploration, opening was revealed fistulous tract that extended from the left external auditory canal down to the anterior border of the sternocleidomastoid muscle. The fistulous tract was noted to run medial to the main truck of the facial nerve (Fig. 1B). Once the facial nerve and its branches had been fully identified, the tract was completely excised with small cuff of external auditory canal skin and cartilage, with primary closure. The branches of facial nerve could be preserved. Facial nerve function was assessed postoperatively by physical examination. The postoperative recovery was uneventful. Histological examination of the excised fistula confirmed that it was lined by squamous epithelium with adnexal structures. A diagnosis of first branchail cleft cyst was made.

\section{Case 2}

A 36-year-old female presented for evaluation of a ruptured 


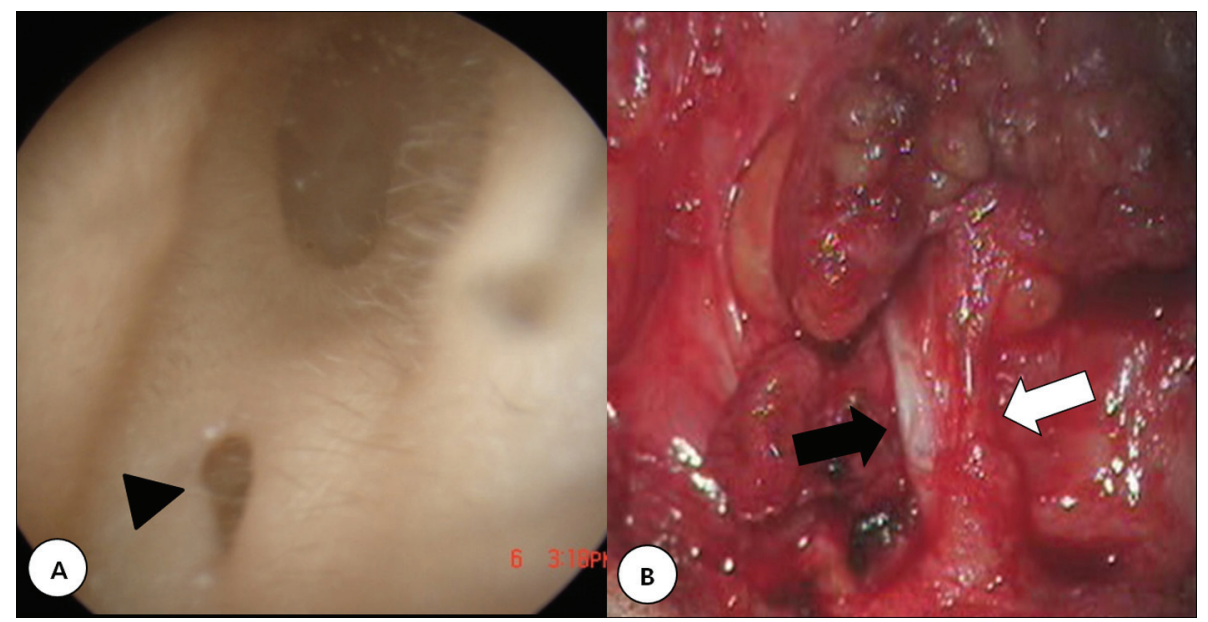

Figure 1. A: A 20-year-old female presented with a sinus opening (arrow head) in the left external auditory canal. B: The track (black arrow) paralleled the normal external auditory canal and terminated deep to the facial nerve (white arrow).

sinus at the anterior border of the right sternocleidomastoid muscle (Fig 2A). The abscess first appeared when she was still a young girl and since then, she had had numerous incision and drainage procedures performed for the recurrent infections. A small opening was identified at the floor of the right external auditory canal. Her tympanic membrane and rest her examination was normal. The patients were prepared, draped, and positioned for a standard parotidectomy. The tract was passing medial to the facial nerve (Fig. 2B). The tract was completely excised with a small cuff of the skin and cartilage.

\section{Discussion}

First branchial anomalies are an uncommon group of lesions that arise from incomplete closure of the ventral portion of the first branchial cleft [1]. These anomalies are closely related to the parotid gland and have variable relations to the facial nerve. The fate of the first branchial apparatus is complete by the sixth or seventh week of development, which means that a first branchial anomaly would be formed by this period [2]. The parotid gland, on the other hand, first appears at the sixth week of development. The facial nerve and its muscles migrate upward between the sixth and eighth week of development. It can be inferred from the embryological patterns that a first branchial cleft anomaly may be found superficial to, deep to, or within the branches of the facial nerve. Reports in the literature suggest most branchial cleft sinuses lie superficial to the facial nerve, while fistulas usually pass deep to the facial nerve $[1,3]$. Sinuses with an external opening situated other than in the external auditory

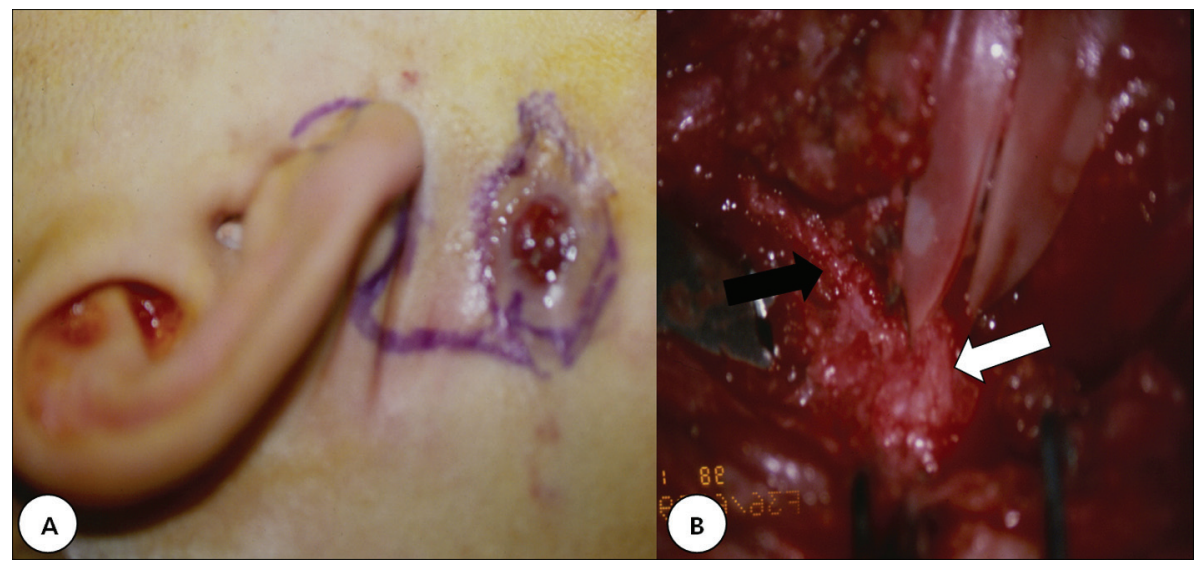

Figure 2. A: 36-year-old female presented with a ruptured sinus at the anterior border of the sternocleidomastoid muscle. B: The fistulous tract (black arrow) was deep to the facial nerve (white arrow) and displaced the nerve laterally. 
meatus are also more likely to be associated with a deep tract. As the nerve courses over the fistula tract, it can lie more inferior than usual, rendering it at risk for trauma during surgery. Hence in order to avoid damage to the facial nerve a conservative superficial parotidectomy with full exposure of the nerve seems to be the logical option [4], together with the use of a facial nerve monitor [5]. D'Souza et al [1] reported that the incidence of temporary facial nerve palsy was $21 \%$ in the patients in whom the nerve was identified and $29 \%$ in the patients in whom the facial nerve was not identified. The incidence of permanent facial paralysis was $1 \%$ in the patients in whom the nerve was identified and $12 \%$ in the patients in whom the nerve was not identified. Identification of the facial nerve trunk at an early stage in the dissection appears to be of critical importance as its relationship cannot be accurately predicted pre-operatively. This approach provides the best chance of ensuring optimal post-operative facial function. The unpredictable relationship of the anomaly with the facial nerve and the frequency of lesions were deep to the nerve place the patient at risk of facial nerve injury during surgery. Therefore, injury is best avoided by identification of the specific lesion type and by understanding its anatomical relationship to the facial nerve. Preoperative computed tomographic scans are very helpful in outlining the relationship of the lesion to the surrounding structures in the neck and in planning the surgical approach $[6,7]$. The computed tomographic scan, however, does not replace careful surgical dissection of the facial nerve. Since the treatment of these anomalies requires a complete resection, and given the risk of facial nerve injury, a wide exposure with complete dissec- tion of the facial nerve is necessary to perform this operation safely. We advocate the use of facial nerve monitoring or nerve stimulator in all cases, especially when inflammatory or postsurgical fibrotic changes make identification of the facial nerve difficult.

\section{References}

1. D'Souza AR, Uppal HS, De R, Zeitoun H. Updating concepts of first branchial cleft defects: a literature review. Int J Pediatr Otorhinolaryngol. 2002;62(2):103109.

2. Ikarashi F, Nakano Y, Nonomura N, Kawana M, Okura T. Clinical features of first branchial cleft anomalies. Am J Otolaryngol. 1996;17(4):233-239.

3. Miller PD, Corcoran M, Hobsley M. Surgical excision of first cleft branchial fistulae. Br J Surg. 1984;71(9):696697.

4. Aimi K, Takino K. Anomaly of the first branchial cleft. Report of a case. Arch Otolaryngol. 1962;75:397-400.

5. Solares CA, Chan J, Koltai PJ. Anatomical variations of the facial nerve in first branchial cleft anomalies. Arch Otolaryngol Head Neck Surg. 2003;129(3):351-355.

6. Nofsinger YC, Tom LW, LaRossa D, Wetmore RF, Handler SD. Periauricular cysts and sinuses. Laryngoscope. 1997;107(7):883-887.

7. Marchioni D, Cuzzola E, Massone F, Ghidini A. Congenital postauricular swelling in a child. Pediatr Dermatol. 2002;19(3):246-249. 